\title{
Survey of prothrombin time in National External Quality Assessment Scheme exercises (1980-87)
}

\author{
L POLLER, D A TABERNER, J M THOMSON, K V DARBY \\ From the United Kingdom Reference Laboratory for Anticoagulant Reagents and Control, Withington Hospital, \\ Manchester
}

SUMmaRY National External Quality Assessment Scheme surveys on the prothrombin time test carried out in hospitals in the United Kingdom have been performed at regular intervals since 1972. Performance has been assessed by comparing observed variability between hospitals with that predicted by a statistical model. The model was based on results from 53 survey plasmas issued between 1980 and 1987. These showed a linear correlation between logarithms of mean and standard deviation of reported ratios. Precision improved until the human brain thromboplastin, Manchester Comparative Reagent, was withdrawn in January 1986. There then followed a pronounced overall deterioration which, by October 1987, had not corrected to the levels achieved by 1985 . When the recent results from 1986-87 were analysed according to Quick test reagent only one reagent (ISI 1·1) showed an improvement in precision. Performance of the other Quick test reagents, all with higher ISI values, had not regained the standards of precision previously achieved by the human brain reagent.

This study was prompted by the need to investigate the effects on precision of the prothrombin time test resulting from the withdrawal of human brain thromboplastin Manchester Comparative Reagent (MCR) in January 1986. Since then, various non-human thromboplastins, mainly of rabbit tissue origin, have been introduced into routine practice in hospitals throughout the United Kingdom. This change has been controversial given that all centres were advised to use International Normalised Ratios (INR). One view contends that rabbit brain thromboplastin has replaced MCR with little difficulty.' On the other hand, a report of the IMLS Haematology Advisory Committee $(1986)^{2}$ reported that many laboratories had observed that their performance in National External Quality Assessment Scheme (NEQAS) exercises seem to have deteriorated significantly since the withdrawal of MCR.

The continuous programme of assessment of the prothrombin time test in the United Kingdom has been conducted on a three monthly basis since 1972 . Reports covering the first five years of the exercises indicated a progressive improvement in performance. ${ }^{34}$ In this study we evaluated the effects on the precision of prothrombin time ratios, determined in successive surveys. Performance over the seven serial

Accepted for publication 16 December 1987 surveys conducted since the withdrawal of human brain MCR was compared with those of the preceding 23 surveys covering the period from July 1980 to October 1985.

\section{Study methods}

To ensure comparability of data between surveys a preliminary analysis was performed to determine whether there was a correlation between the prothrombin ratio of survey plasmas and the standard deviation (SD). This was shown by the statistical method of ordinary least squares fit and was taken into account in the index of performance described below.

Thirty NEQAS prothrombin time surveys had been conducted at intervals of three months between July 1980 (Survey 30) and October 1987 (Survey 59). Results from surveys before 1980 have not been considered in this report for two main reasons: the number of participants had not reached a stable level as many new participants were entering the scheme, and the nature of the test plasmas was also more variable.

In each survey about 400 participants reported results on lyophilised test plasmas. A total of 53 test plasmas obtained by plasmapheresis from patients receiving coumarin treatment were included. Survey 40 was not included in the analysis as it was not 
possible to quantify the coumarin defect in ratio terms, in view of severe overdosing. The number of plasmas tested in each exercise varied between one and three; they represented different intensities of anticoagulation and all were single donations. Multiple samples were issued in seven of the surveys before the withdrawal of MCR and the last four included three coumarin plasmas. Six of the seven surveys since January 1986 contained three coumarin plasma samples.

On each occasion, participants were requested to report results as prothrombin times and prothrombin ratios. Prothrombin ratios with MCR had been designated British Ratios by the British Society for Haematology. ${ }^{5}$ From Survey 49 (April 1985) onwards, results were reported as INR. This was introduced to standardise prothrombin time testing in oral anticoagulant control and the method of reporting. ${ }^{6-8}$ INR are ratios which, it is estimated, would have been obtained with the World Health Organisation first primary reference preparation for thromboplastin, 67/ 40. These are virtually identical with the British ratio used previously, as the International Sensitivity Index of MCR was invariably close to $1 \cdot 0$.

\section{Results}

A linear relation between the severity of the coagulation defect and the SD was found when the means and SD for the test plasmas were plotted on a double logarithmic scale (fig 1). This was estimated by a regression model: natural logarithm of the SD of the

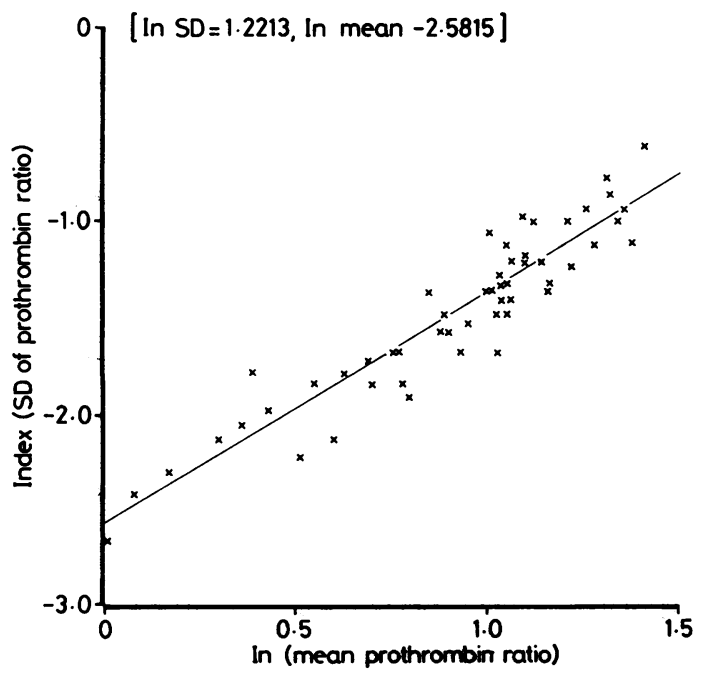

Fig 1 Linear relation between natural $\log$ In of SD of prothrombin time ratio and that of mean prothrombin ratio obtained from results from NEQAS test plasmas.
Table 1 Expected SD given different mean levels of coagulation defect*

\begin{tabular}{|c|c|c|c|}
\hline $\begin{array}{l}\text { Mean } \\
\text { standardised } \\
\text { prothrombin } \\
\text { ratio }\end{array}$ & $\begin{array}{l}\text { Expected } \\
S D\end{array}$ & $\begin{array}{l}\text { Expected } \\
C V \% \\
\text { (Expected } S D \\
\div \text { mean })\end{array}$ & $\begin{array}{l}95 \% \text { confidence } \\
\text { interval for } \mathrm{CV} \%\end{array}$ \\
\hline $\begin{array}{l}1.0 \\
1.5 \\
2.0 \\
2.5 \\
3.0 \\
3.5 \\
4.0 \\
4.5 \\
5.0\end{array}$ & $\begin{array}{l}0.08 \\
0.12 \\
0.18 \\
0.23 \\
0.29 \\
0.35 \\
0.41 \\
0.47 \\
0.54\end{array}$ & $\begin{array}{r}8.0 \\
8 \cdot 3 \\
8.8 \\
9 \cdot 3 \\
9.6 \\
10.0 \\
10 \cdot 3 \\
10.6 \\
10.8\end{array}$ & $\begin{array}{l}6 \cdot 7-8 \cdot 6 \\
7 \cdot 6-9 \cdot 0 \\
8 \cdot 4-9 \cdot 3 \\
8 \cdot 9-9 \cdot 7 \\
9 \cdot 2-10 \cdot 2 \\
9 \cdot 4-10 \cdot 6 \\
9 \cdot 5-11 \cdot 1 \\
9 \cdot 6-11 \cdot 5 \\
9 \cdot 8-12 \cdot 0\end{array}$ \\
\hline
\end{tabular}

${ }^{*}$ Calculations are based on the model $\ln (\mathrm{SD})=1.2213 \times \ln ($ mean ratio) $-2 \cdot 5815$.

Table 2 Index of performance in NEQAS exercises expressed as \% of expected SD. Means for each survey are given in parentheses.

\begin{tabular}{|c|c|c|c|c|c|c|c|c|}
\hline \multirow[b]{2}{*}{$\begin{array}{l}\text { Survey } \\
\text { No } \\
30 \\
31 \\
32 \\
33 \\
34 \\
35 \\
36 \\
37 \\
38 \\
39 \\
41 \\
42 \\
43 \\
44 \\
45\end{array}$} & \multicolumn{3}{|c|}{$\begin{array}{l}\text { Plasma } \\
\text { performance } \\
\text { index }\end{array}$} & \multirow[b]{2}{*}{$\begin{array}{l}\text { Survey } \\
\text { No } \\
46 \\
47 \\
48 \\
49 \\
50 \\
51 \\
52 \\
53 \\
54 \\
55 \\
56 \\
57 \\
58 \\
59\end{array}$} & \multicolumn{4}{|c|}{$\begin{array}{l}\text { Plasma } \\
\text { performance } \\
\text { index }\end{array}$} \\
\hline & $\begin{array}{r}100 \\
108 \\
99 \\
92 \\
85 \\
94 \\
90 \\
120 \\
102 \\
95 \\
107 \\
86 \\
91 \\
86 \\
83\end{array}$ & $\begin{array}{l}102 \\
104\end{array}$ & $\begin{array}{r}(105) \\
(97)\end{array}$ & & $\begin{array}{r}78 \\
86 \\
82 \\
92 \\
72 \\
98 \\
74 \\
108 \\
124 \\
100 \\
117 \\
99 \\
100 \\
108\end{array}$ & $\begin{array}{r}92 \\
76 \\
99 \\
87 \\
122 \\
140 \\
108 \\
103 \\
114 \\
116 \\
111\end{array}$ & $\begin{array}{r}110 \\
91 \\
103 \\
81 \\
135 \\
\\
130 \\
109\end{array}$ & $\begin{array}{r}(97) \\
(81) \\
(100) \\
(81) \\
(121) \\
(132) \\
(112) \\
(109) \\
(106) \\
(113) \\
(111)\end{array}$ \\
\hline
\end{tabular}

prothrombin ratios is equal to the natural logarithm of the mean prothrombin ratio $\times 1 \cdot 2213-2.5815$. This gave an excellent fit to the data ( $p$ value of the $F$ test $<$ 0.01 ). The statistical assumptions of normality of deviations from the line and stability of variance about the line were met.

Table 1 gives the expected SD and coefficient of variation (CV) values predicted by the model for different levels of coagulation defect. These are based on average performance over seven years with NEQAS plasmas that had INRs between 1.01 and 4.08. The performance index is defined as the deviation of each observed SD with an individual test plasma from the model-that is, the deviation from the average performance after correction for the level of coagulation defect (fig 2). The $100 \%$ figure represents the mean SD of the results over the seven year period. Those above $100 \%$ represent a deterioration in SD; results less than $100 \%$ denote improvement.

Table 2 gives the performance index for each 


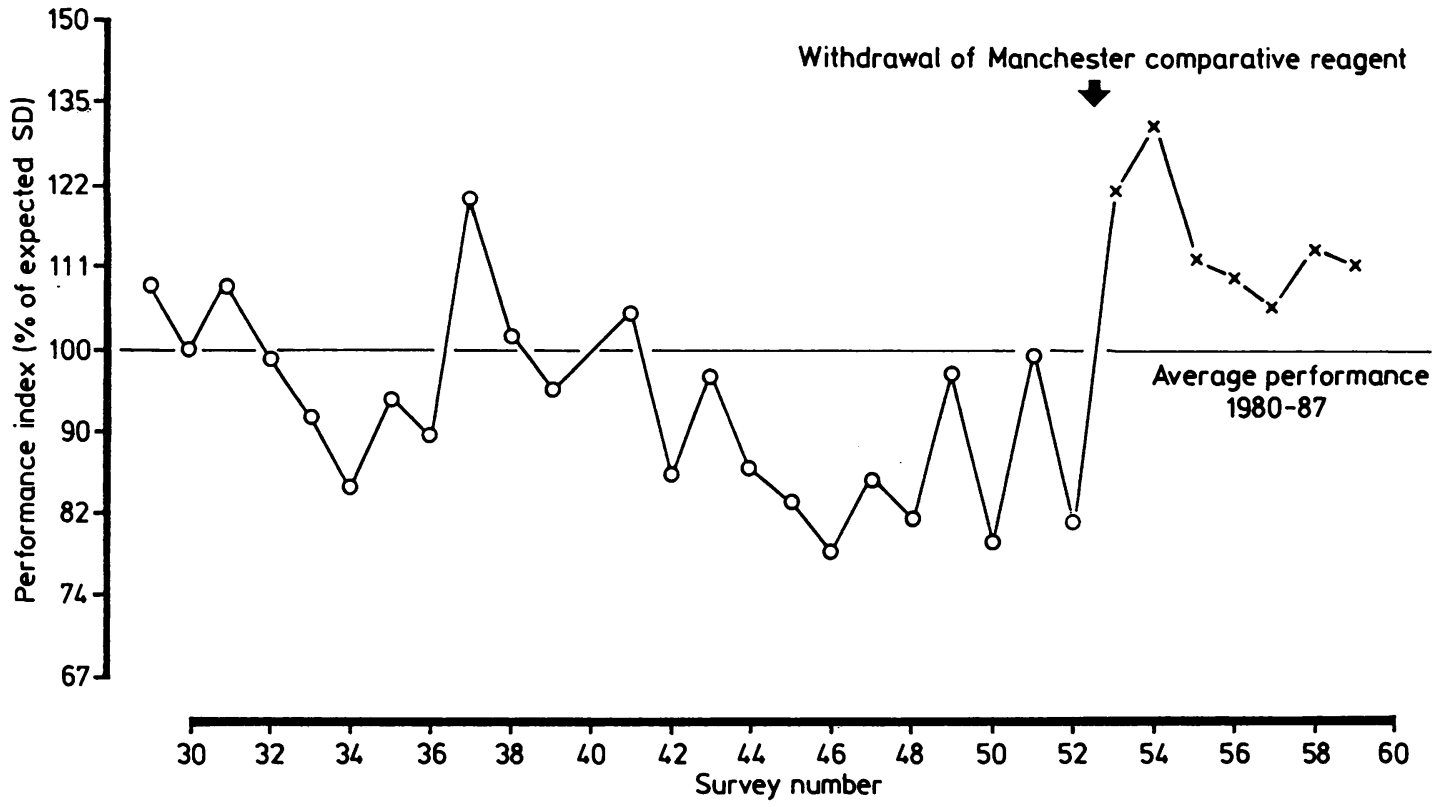

Fig 2 Performance indices of NEQAS surveys (30-59) for prothrombin time test showing association between average performance and effects of withdrawal of $M C R$.

plasma, expressed as a percentage of the expected level of variation. Indices for surveys in which more than one plasma were tested are also provided. In fig 2 the performance indices are plotted over the seven year period, showing a trend to lower than average SD until survey 53 which followed the withdrawal of MCR. In

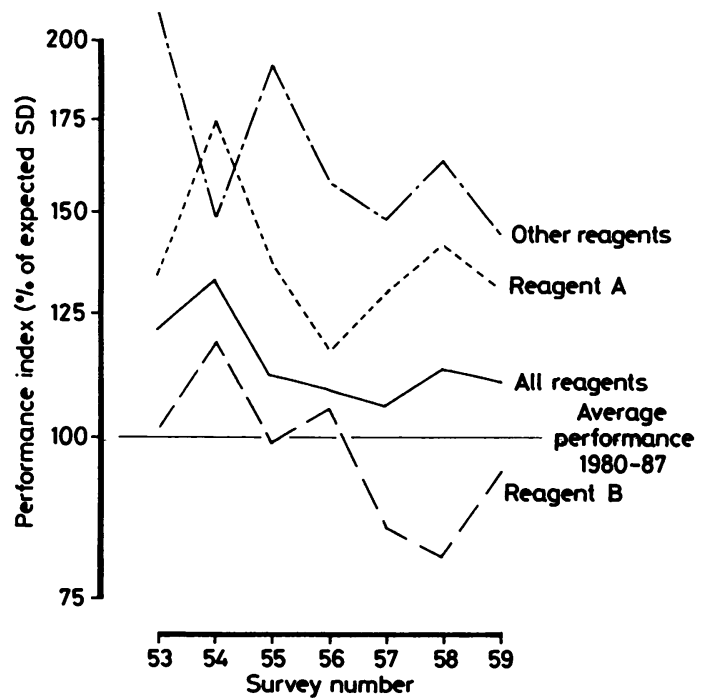

Fig 3 Performance indices of NEQAS surveys (53-59) with various thromboplastin reagents. this survey there was a sudden increase in variability. There has been a partial recovery since, but variability has remained high and was $11 \%$ above average in the last survey considered (59). Before survey 53 in April 1986 MCR was used almost exclusively in hospitals in the United Kingdom. Since then a variety of commercial Quick test thromboplastins have been in general use.

Only two reagents (coded A and B) were used by enough participants for separate analysis to be made (more than 20 returns). Reagents used in smaller numbers were classified together as "other reagents". Seventy five per cent of participants used reagent B in surveys 53-59. When the results with this reagent were compared with overall performance a steady improvement was seen (fig 3). In surveys 57-59 this reagent gave a level of variability below the overall average for the seven year period. In contrast, reagent A, showed a deterioration in performance compared with the previous results with MCR and the average performance of all thromboplastin reagents over the seven years. Results with "other reagents" were consistently more variable, the SD ranging between 30 and $80 \%$ greater than the average provided by the model.

\section{Discussion}

A serial programme of external quality assessment surveys in prothrombin time testing has been conduc- 
ted in the United Kingdom since 1972. Between 1972 and 1973 the prothrombin time test showed considerable variation between hospitals with only a slight improvement over successive exercises. ${ }^{3}$ Further analysis of data extending up to 1977 showed evidence of improvement in performance. ${ }^{4}$

The present study clarifies the situation which has arisen following the withdrawal of the human brain MCR, used routinely in $95 \%$ of United Kingdom hospitals up to January 1986. It provides evidence of the subsequent deterioration of overall precision and the long term effects of the change in reagents on the prothrombin time test.

An increase in the variability of standardised prothrombin ratios (British ratio or INR) between hospitals was observed. The variability was quantified by deriving a performance index for each NEQAS test plasma, which was independent of the severity of the coagulation defect. The derivation of standardised performance indices was based on the linear relation observed between logarithms of mean ratios and corresponding SD.

Taking into account the degree of coagulation defect for the individual plasmas, the overall performance in NEQAS exercises only marginally improved after the deterioration observed following the withdrawal of MCR. A sustained improvement in performance has, however, been observed with one reagent (B), which by late 1987 gave similar precision to that previously achieved with human brain MCR. In contrast, the remaining $25 \%$ of hospitals have continued to give results showing relatively poor precision without consistent evidence of reduction of SD.

The responsiveness of thromboplastin reagents to the coumarin defect, which is quantified by the International Sensitivity Index, may be an important factor in the variability of INR which ensued. The
Quick test thromboplastin reagents of rabbit origin, apart from reagent $B$, have an ISI of 1.4 or more, whereas reagent $B$ has an ISI of $1 \cdot 1$. A possible explanation may therefore relate to the ISI value of the thromboplastin. In theory, for an ISI of more than 1.0 , a fixed error in prothrombin ratio will represent a larger error in the corresponding INR. This is quantitatively dependent on the ISI; a higher ISI will give greater increases in variability.

This study shows that the overall performance in prothrombin time testing compares unfavourably with the situation at the end of 1985 when human brain MCR was withdrawn, but this is accounted for by the deterioration in performance by a minority of participants using reagents with higher ISI values.

\section{References}

1 Anonymous. Oral anticoagulant control [Editorial]. Lancet 1987;ii:488-9.

2 Recommendations for the Laboratory Determination of the INR. Report of the Haematology Advisory Committee. IMLS Gazette 1986;xxx:603-5.

3 Leck I, Gowland E, Poller L. The variability of measurements of the prothrombin time ratio in the National Quality Control Trials: a follow-up study. Br J Haematol 1974;28:601-12.

4 Poller L, Thomson JM, Yee KF. Quality control trials of prothrombin time: An assessment of the performance in serial studies. J Clin Pathol 1979;32:251-3.

5 Poller L. The British comparative thromboplastin: the use of the national thromboplastin reagent for uniformity of laboratory control of oral anticoagulants and expression of results. London: Association of Clinical Pathologists/BMA, 1970:B/S 71.

6 Besselaar AMHP van den, Loeliger EA, Poller L, Thomson JM, Tomenson JA. Standardisation of oral anticoagulant treatment. Br Med J 1984;288:486-7.

7 ICTH/ICSH. Prothrombin time standardisation: report of the expert panel on oral anticoagulant control. Thromb Haemostas 1979;42:1073-114.

$8 \mathrm{ICTH} / \mathrm{ICSH}$. Recommendations for reporting prothrombin time in oral anticoagulant control. J Clin Pathol 1985;38:133-4. 